\title{
Celiprolol Double-Peak Occurrence and Gastric Motility: Nonlinear Mixed Effects Modeling of Bioavailability Data Obtained in Dogs
}

\author{
Elke Lipka, 1,3,4 I-Der Lee, ${ }^{1,5}$ Peter Langguth, ${ }^{1,2}$ Hildegard Spahn- \\ Langguth, ${ }^{3}$ Ernst Mutschler, ${ }^{3}$ and Gordon L. Amidon ${ }^{1,6}$
}

Received March 2, 1995-Final August 24, 1995

\begin{abstract}
Investigation of the underlying mechanism leading to inter-and intrasubject variations in the plasma concentration-time profiles of drugs (1) can considerably benefit rational drug therapy. The significant effect of gastric emptying on the rate and extent of celiprolol absorption and its role with respect to double-peak formation was demonstrated in the present study. In four dogs racemic celiprolol was dosed perorally in a crossover design during four different phases of the fasted-state gastric cycle and gastric motility was recorded simultaneously using a manometric measurement system. Intravenous doses were also given to obtain disposition and bioavailability parameters. The blood samples were assayed by a stereoselective HPLC method (2). The time to onset of the active phase of the gastric cycle showed an excellent correlation with the time to celiprolol peak concentration. Furthermore, bioavailability was increased when celiprolol was administered during the active phase. Double peaks were observed when the first active phase was relatively short, suggesting that a portion of the drug remained in the stomach until the next active phase. Population pharmacokinetic modeling of the data with a two-compartment open model with two lag times incorporating the motility data confirmed the effect of time to gastric emptying on the variability of the oral pharmacokinetics of celiprolol. The fasted-state motility phases determine the rate and extent of celiprolol absorption and influence the occurrence of double peaks. Peak plasma levels of celiprolol exhibit less variability if lag times, and therefore gastric emptying times, are taken into consideration.
\end{abstract}

KEYWORDS: gastric motility; celiprolol; NONMEM; double-peak phenomenon; variable oral pharmacokinetics; nonlinear oral bioavailability; gastric emptying.

This work was supported by FDA-01462 and in part by the Dr. Robert Pfleger-Stiftung (Bamberg, Germany).

'College of Pharmacy, University of Michigan, Ann Arbor, Michigan 48109.

${ }^{2}$ Department of Pharmacy, ETH, CH-8093 Zuerich, Switzerland.

${ }^{3}$ Department of Pharmacology, Johann Wolfgang Goethe-University, Biocenter, Niederursel, Marie-Curie-Strasse 9, Geb. N260, D-60439 Frankfurt/Main, Germany.

4Present address: TSRL Inc., 540 Avis Drive, Suite A, Ann Arbor, Michigan 48108.

${ }^{5}$ Present address: Clinical Pharmacology Department, Hoechst-Roussel Pharmaceuticals, Route 202-206, Somerville, New Jersey 08876.

${ }^{6}$ To whom correspondence should be addressed. 


\section{INTRODUCTION}

Celiprolol, 3-[3-acetyl-4-[3-t-(butyl-amino)-2-hydroxy-propoxy]-phenyl]1,1-diethylurea hydrochloride (MW $414 \mathrm{~g} / \mathrm{mol}$ ), is a hydrophilic $\beta$-adrenoceptor antagonist with a $\mathrm{p} k_{\mathrm{a}}$ of 9.7 . It is highly soluble in water $(176 \mathrm{mg} / \mathrm{ml})$ and the logarithm of the octanol/water partition coefficient $\log P$ amounts to 0.13 (3), which is comparable to that of, e.g., atenolol $(\log P, 0.2)$. The chemical structure of the drug is illustrated in Fig. 1. (4).

The parmacokinetic profile of celiprolol shows two unusual and interesting characteristics. The plasma concentration-time curve frequency exhibits two peaks, and the bioavailability is low and increases with dose (5). The drug is not metabolized to any significant extent and is eliminated equally via urine and bile. Nonlinear increase in bioavailability after oral drug administration has been reported for other drugs like penicillamine (6), as well as pafenolol $(7,8)$ and nadolol $(9)$, which exhibit a similar pharmacokinetic profile as celiprolol. These observations appear to be very complex in origin, since they could be due to limited liver extraction capacity, saturable renal excretion, or a dose-dependent uptake from the gastrointestinal tract. However, since celiprolol shows dose linearity after intravenous (iv) administration in humans (10) as well as in preliminary dog studies (data not shown), and since a dose-dependence of the terminal half-life was neither detected following iv nor following po dosage in dogs, a nonlinear absorption process appears to be the most likely explanation.

Nadolol has been shown to form stable micellar complexes with bile salts, which leads to a decrease in thermodynamic activity and, therefore, to an increased oral clearance and bioavailability with low doses $(11,12)$. Lennernäs and Regardh (13) proposed the same mechanism for pafenolol. Furthermore, they regarded bile salt complexation as a possible explanation for the occurrence of double peaks. Since bile salts are actively reabsorbed in the distal ileum, the drug may be released from the complex due to

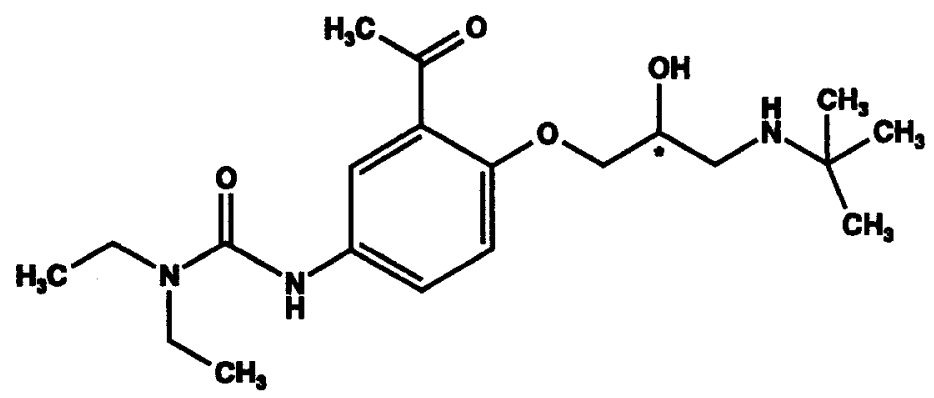

FIG. 1. Chemical structure of celiprolol (* chiral centre). 
equilibrium reaction and thereafter be absorbed, leading to a second peak in the plasma concentration-time profile.

Another hypothesis for explaining the double-peak phenomenon is gastric emptying-limited absorption kinetics. In general, the main factors influencing drug absorption include the rate of disintegration of the dosage form, the dissolution rate, and the intestinal wall permeability of the compound (14). For drugs that are not restrained by any of the above factors, gastric emptying can become the rate-limiting step in absorption. During the fasted state the gastrointestinal tract shows a cyclic pattern of contractions, consisting of quiescent phases (Phase I) without contractions of 1 to $3 \mathrm{hr}$ followed by active phases with intense contractions (Phase II/III) of about 5-30 min. Gastric emptying is mainly associated with the active phase of the gastric cycle. Variations in gastric emptying pattern may therefore lead to variations in peak blood levels and times to peak. Furthermore, incomplete emptying either during the initial first-order emptying or the first active phase could result in a two-phase absorption with a portion of the drug remaining in the stomach until the next active phase occurs.

Double or multiple peaks in the concentration-time profile and/or variations in the invasion profile have been reported for a series of drugs including acetaminophen (15), cimetidine (16), ranitidine (17), furosemide (18), flurbiprofen (19), pafenolol (20), veralapride (21), ciclotropium (22), etacrynic acid (23), ibuprofen (24), tranylcypromine (25), and celiprolol (26). However, in only a few cases the available data could provide an explanation. Flurbiprofen, for example, has shown to be most likely gastric emptying controlled in its absorption profile (19). Recent studies by Artursson et al. (27) in Caco-2 cells suggest that an active intestinal secretion (exsorption) of celiprolol in the proximal part of the intestine could be responsible for the nonlinear bioavailability. These authors hypothesized that this kinetic process may as well be a causative factor for the double peaks. Facing this variety of hypotheses, a thorough investigation of the absorption mechanism of celiprolol is therefore necessary to explain nonlinear bioavailability and double-peak phenomenon.

\section{MATERIALS AND METHODS}

\section{Dog Studies}

Four dogs (two female mongrels, one male and one female beagle) were included in the present study. Prior to each experiment, the dogs were fasted for $18 \mathrm{hr}$ with free access to water.

Recordings of the gastric motor activity were obtained using a pneumohydraulic manometric system, which consisted of a flexible catheter 

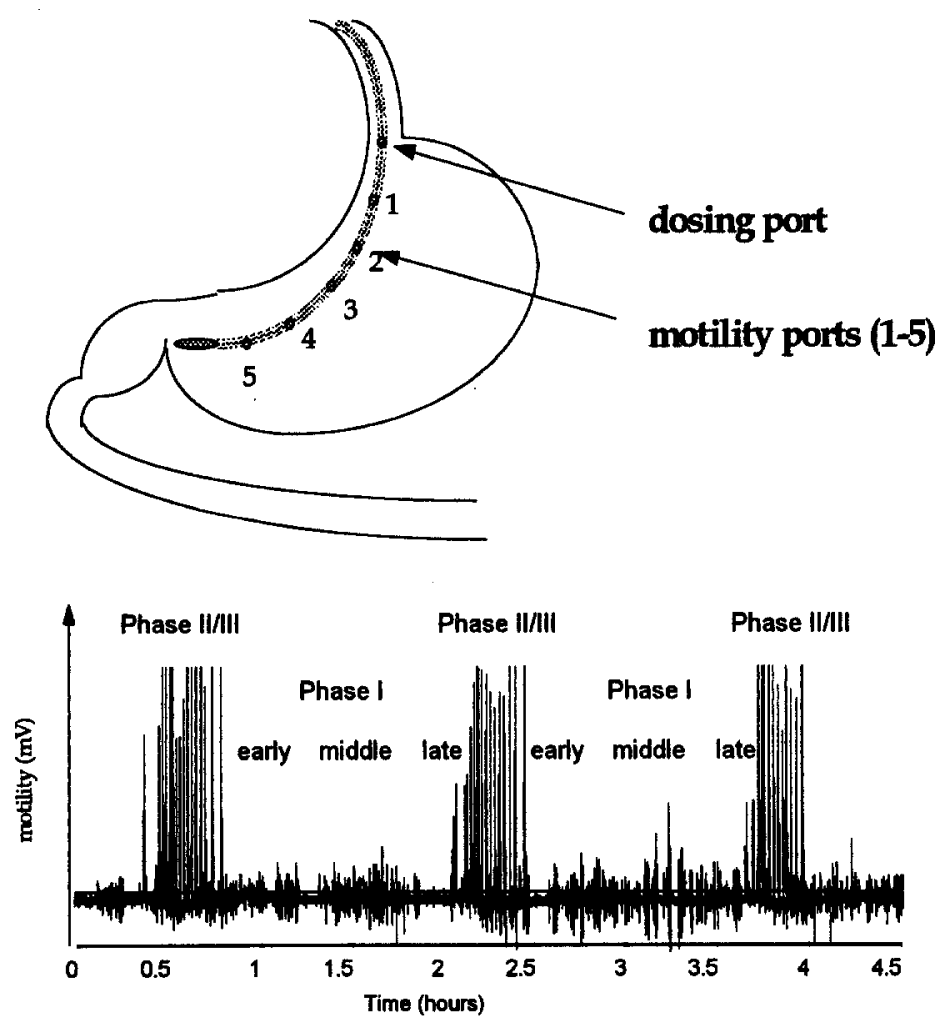

FIG. 2. Placement of the multilumen catheter in the stomach and representative fasted-state motility cycle

containing six motility probes. After swallowing of the oral catheter by the dog, at least two recording sites were placed in the proximal and distal antrum. Distilled water was infused through these ports at a rate of $1.5 \mathrm{ml} /$ min. Stomach contractions interrupted the water flow by closing the openings in the catheter, resulting in a back pressure along the tube (Fig. 2.). Strain gauge transducers transformed the intraluminal pressure into electrical signals, which were then printed on a chart recorder and saved on a computer for further analysis.

Motility was recorded continuously for $4 \mathrm{hr}$ after drug administration. Oral doses of $150 \mathrm{mg}$ racemic celiprolol in $30 \mathrm{ml}$ water were given as a solution in a crossover design during four different phases of the gastric cycle: early Phase I, middle of Phase I, late Phase I, and Phase III. Intravenous doses of $50 \mathrm{mg}$ racemic celiprolol were also given to obtain disposition and bioavailability parameters. Blood samples were obtained from an 
indwelling heparin lock catheter, which was placed in the cephalic vein of the front leg, prior to dosing and 15, 30, 45, 60, 75, 90, 120, 150, 180, 240, 300 , and $360 \mathrm{~min}$ after dosing for the po study and prior to dosing and 2 , $5,10,20,30,45,60,75,90,120,150,180,240,300$, and $360 \mathrm{~min}$ after dosing for the iv study. The samples were centrifuged and stored at $-20^{\circ} \mathrm{C}$ until analyzed.

\section{Analytical Methods}

Plasma samples were analyzed using a enantiospecific HPLC assay described previously (2).

\section{Data Analysis}

Noncompartmental analysis was performed employing standard definitions and calculation procedures. The motility data were analyzed utilizing STATISTICA (release 4.1).

Population pharmacokinetic parameters were obtained with a twocompartment open model that included two input (depot) compartments with two lag times, into which the gastrointestinal motility data were incorporated (Fig. 3). The first depot compartment represents the fraction of the dose that is emptied into the intestine with occurrence of the first Phase II/III of the gastric cycle. With the following Phase II/III, the remaining portion of the dose (in the second depot compartment) is released from

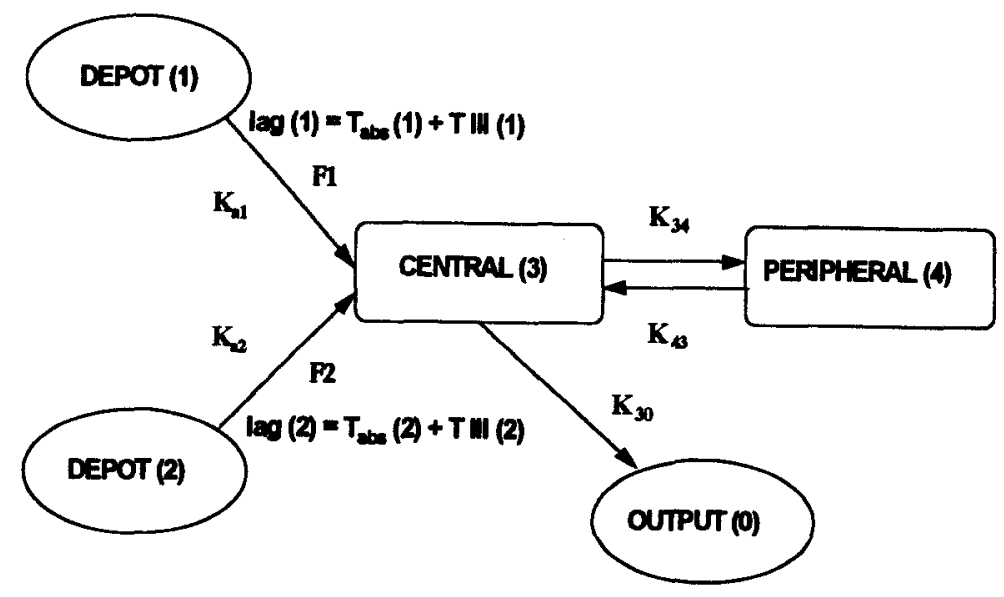

FIG. 3. The two-compartment open model incorporating gastric motility data and two lag times for different dose fractions. ( $\mathrm{Lag}=$ total lag time following po dosage $=T_{\text {abs }}+T$ III $; T$ III : time to onset of Phase II/III; $T_{\text {abs }}$ : effective absorption lag time for celiprolol from the intestine into the systemic circulation.) 
the stomach. In both cases, a lag time is observed that may be divided into the time to Phase II/III ( $T$ III), assuming this is the time when the drug is emptied from the stomach, and the time to absorption $\left(T_{\text {abs }}\right)$. The rationale for incorporating the time to Phase $\mathrm{III}$ into the model was to reduce the variability in the onset of absorption, which was due to variable gastric emptying. Therefore, the parameter to be estimated was not the lag time but the actual absorption time ( $T_{\text {abs }}$ ) required for the drug to enter the central compartment after leaving the stomach. Thus, two bioavailability fractions, $F I+F 2$, are obtained from the two absorption events. The central compartment represents the blood, whereas the peripheral compartment represents the tissue. The model is characterized by a set of first-order linear differential equations

$$
\begin{array}{rlrl}
\frac{d A}{d t} & =0 & & \text { for } t<T \text { III (1) } \\
\frac{d A 1}{d t}=-k_{\mathrm{a} 1} \times A 1 & & \text { for } t>T \text { III (1) } \\
\frac{d A 2}{d t}=-k_{\mathrm{a} 2} \times A 2 & \text { for } t>T \text { III (2) } \\
\frac{d A 3}{d t}=k_{\mathrm{a} 1} \times A 1+k_{\mathrm{a} 2} \times A 2-k_{34} \times A 3+k_{43} \times A 4-k_{30} \times A 3 \\
\frac{d A 4}{d t}=k_{34} \times A 3-k_{43} \times A 4 &
\end{array}
$$

where $k_{\mathrm{a} 1}$ and $k_{\mathrm{a} 2}$ are the rate constants for the drug entering the central compartment after lag time 1 and lag time 2 , respectively. The distribution into the peripheral compartment is represented by $k_{34}$, and the velocity for redistribution into the central compartment is determined by $k_{43}$. The rate constant for elimination from the central compartment is $k_{30} . A 1, A 2, A 3$, and $A 4$ are the amounts of drug in the depot compartments 1 and 2, central compartment 3, and peripheral compartment 4, respectively. The parameters to be estimated employing this model are $k_{\mathrm{a} 1}, k_{\mathrm{a} 2}, k_{34}, k_{43}, k_{30}, t_{\mathrm{abs} 1}, t_{\mathrm{abs} 2}$, $F 1$, and $F 2$. Additionally, the volume of the central compartment (V3) has to be estimated in order to convert blood concentrations into amounts in the four compartments. The computer program NONMEM 77 Version IV Level 1 (written in ANSI FORTRAN 77) was used to estimate the pharmacokinetic parameters. NONMEM (Non-linear Mixed Effects Model) is designed to fit general statistical (nonlinear) regression type models to data (28), offering two major advantages when compared to other nonlinear regression programs: first, it is able to incorporate fixed effects, mainly physiological parameters that vary among subjects, into the pharmacokinetic 
model. Second, it can pool data simultaneously from different individuals. Intraindividual and interindividual variations can therefore be separated and quantified.

Data from the four dogs obtained in the study described above were analyzed. The total set of data contained four iv studies and 16 po studies. In the first step, the model parameters were fitted to the individual iv data sets, subsequently, each po set was modeled simultaneously with the corresponding iv data, resulting in an individual fit of all po and iv data within one dog. Intraindividual variability was obtained from the difference between the predicted and the observed data. Finally, parameters of the individual model were fitted to the complete data set.

After incorporating population error parameters for bioavailability into the model, the four iv data sets and subsequently the entire data pool were fitted to obtain the average pharmacokinetic profile and the interindividual error. Although a limited data set of 20 experiments was analyzed by the NONMEM, which might be somewhat small to be considered a population, the NONMEM terminology for the population parameters is used throughout the rest of the manuscript.

\section{RESULTS}

In the dog only Phase I (quiescent phase) and Phase II/III (active phase) of the gastric cycle can be clearly distinguished, with mean values for the duration of the two phases being $57.2 \pm 8.2$ and $19.4 \pm 4.5 \mathrm{~min}$, respectively (Fig. 4). These time frames are in good agreement with data obtained employing electrical measurement of the action potentials in dogs (29), as well as with data reported for humans (30).

Examples of two individual plasma profiles, dosed in the quiescent phase, together with the corresponding motility data are shown in Fig. 5(a and b). Significant lag times were observed with all experiments where the drug was administered in Phase I, suggesting that celiprolol is not absorbed from the stomach and can only reach the absorption site after gastric emptying has taken place. Highest variations among the lag times were observed when the drug was administered during the early quiescent phase, since the emptying of celiprolol depends on the timing of dosing relative to the occurrence of the next active phase (Fig. 6a). Variations in the intravenous data are relatively small by comparison (Fig. 6b), suggesting that varying factors during the absorption process determine the variability in the oral pharmacokinetic profile of celiprolol. Biphasic absorption, resulting in double peaks or shouldering in the plasma concentration-time profile, was usually observed when the duration of the active phase was relatively short 


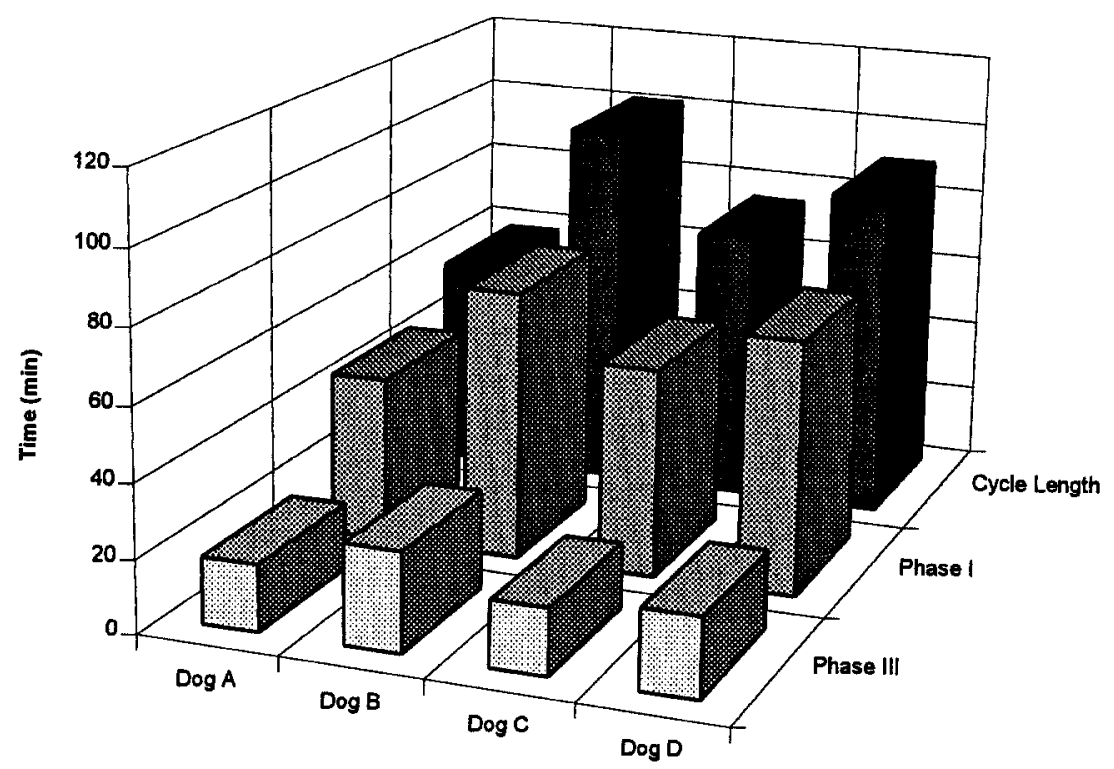

FIG. 4. The duration of the different phases of the gastric cycle in the fasted state for four dogs.

$(<10-15 \mathrm{~min})$. An excellent correlation $(r=.97)$ was obtained between the time to gastric emptying and the time to peak concentration (including the time to the second active phase when an additional plasma peak was observed), showing a linear relationship with a slope close to 1 (Fig. 7). Therefore, the intercept of the correlation line $(b=0.55 \mathrm{hr})$ may be interpreted as the actual absorption time, needed for the drug to enter the systemic circulation after reaching the intestine. The double-peak frequency observed was approximately $75 \%$ when all data sets showing either a clearly pronounced second peak or shouldering in the concentration-time profile (that was above the assay precision limit) were included.

Interestingly, bioavailability was significantly increased and less variable when celiprolol was administered during Phase II/III (Fig. 8). This is most likely due to the better mixing effect in the intestine and/or less dilution by the gastrointestinal fluids and the water infused by the motility equipment. The resulting mean pharmacokinetic parameters from four studies in each dog, given in Table I, confirm again the high inter- and intrasubject variability regarding $t_{\max }, C_{\max }$, and bioavailability. However, due to the strong correlation between time to Phase III and $t_{\max }$, gastric emptying can explain the variations in the time to the onset of absorption. Peak concentration and rate of absorption may still vary, especially when the drug is dosed in 
a)

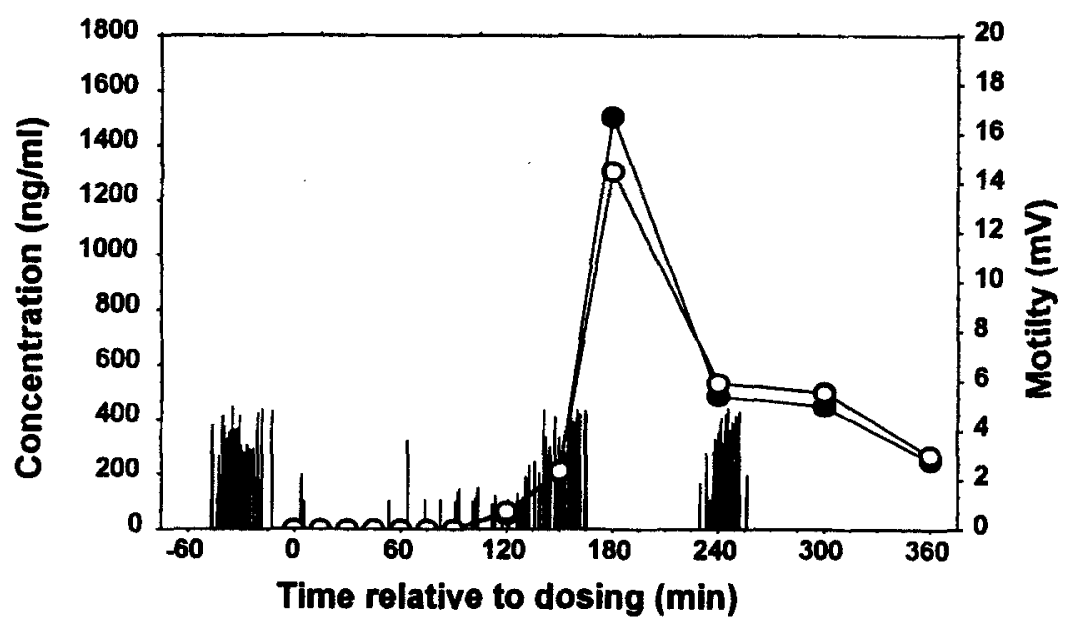

b)

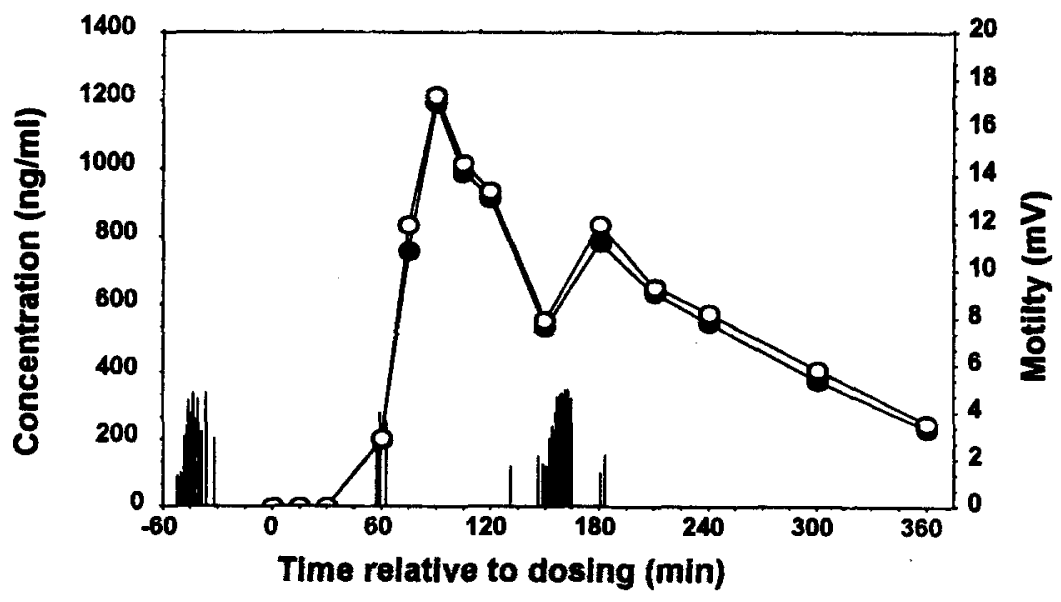

FIG. 5. Plasma concentration-time profile of $150 \mathrm{mg}$ racemic celiprolol- $\mathrm{HCl}$ po dosed in (a) early Phase 1, (b) middle of Phase I with the corresponding motility data (closed circles $=$ S-celiprolol, open circles $=$ R-celiprolol) . 
a)

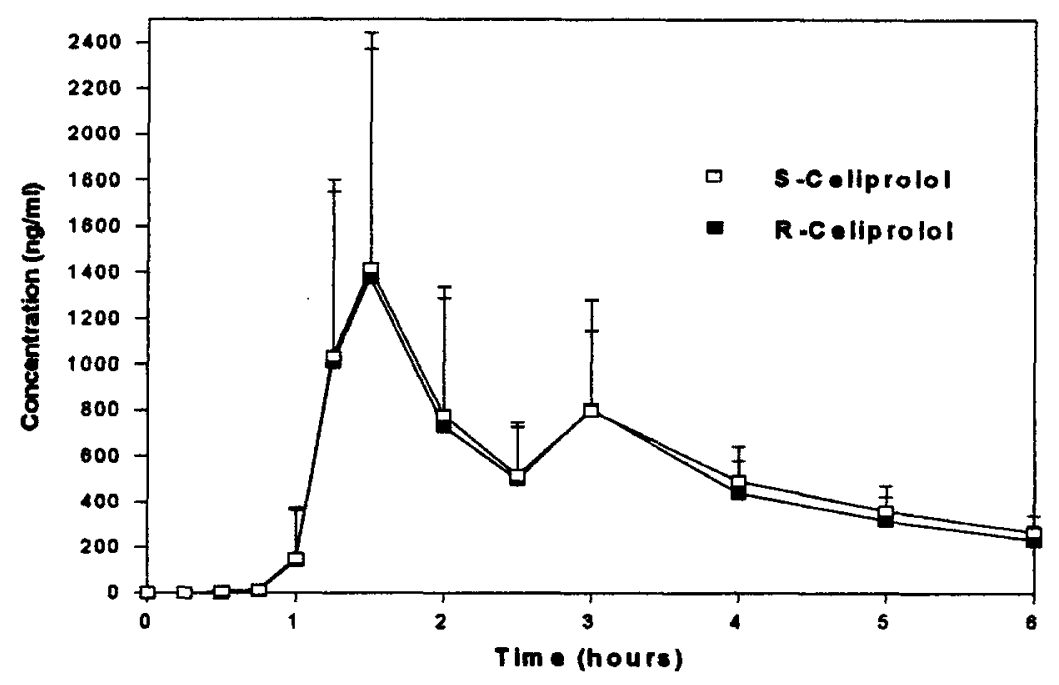

b)

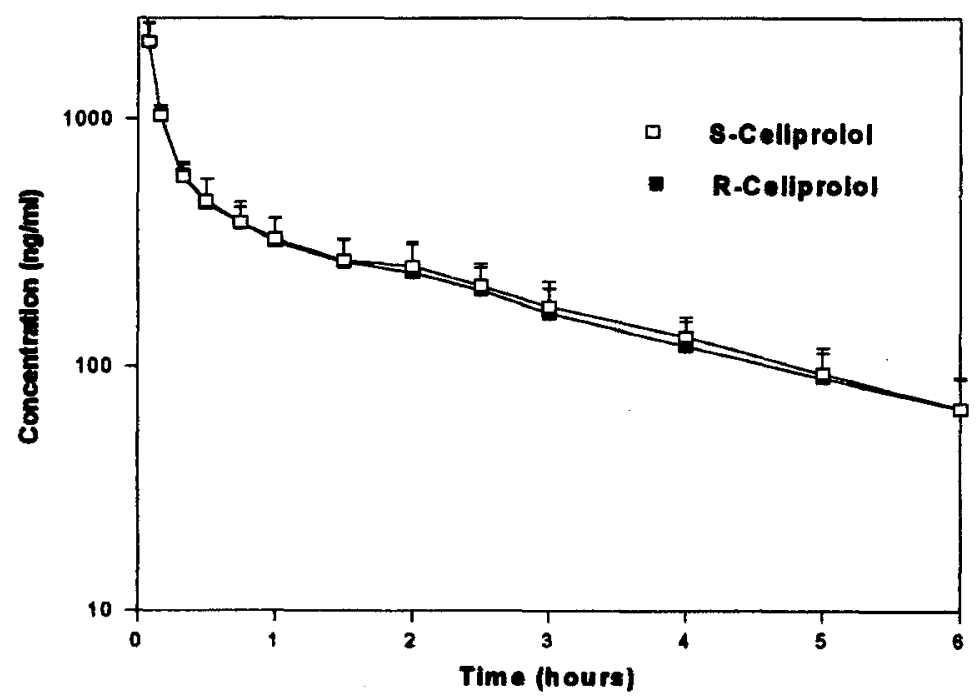

FIG. 6. Mean plasma concentration-time profiles of (a) $150 \mathrm{mg}$ racemic celiprolol$\mathrm{HCl}$ dosed orally in early Phase I $(n=4)$ and (b) $50 \mathrm{mg}$ racemic celiprolol- $\mathrm{HCl}$ after intravenous administration $(n=4)$. 


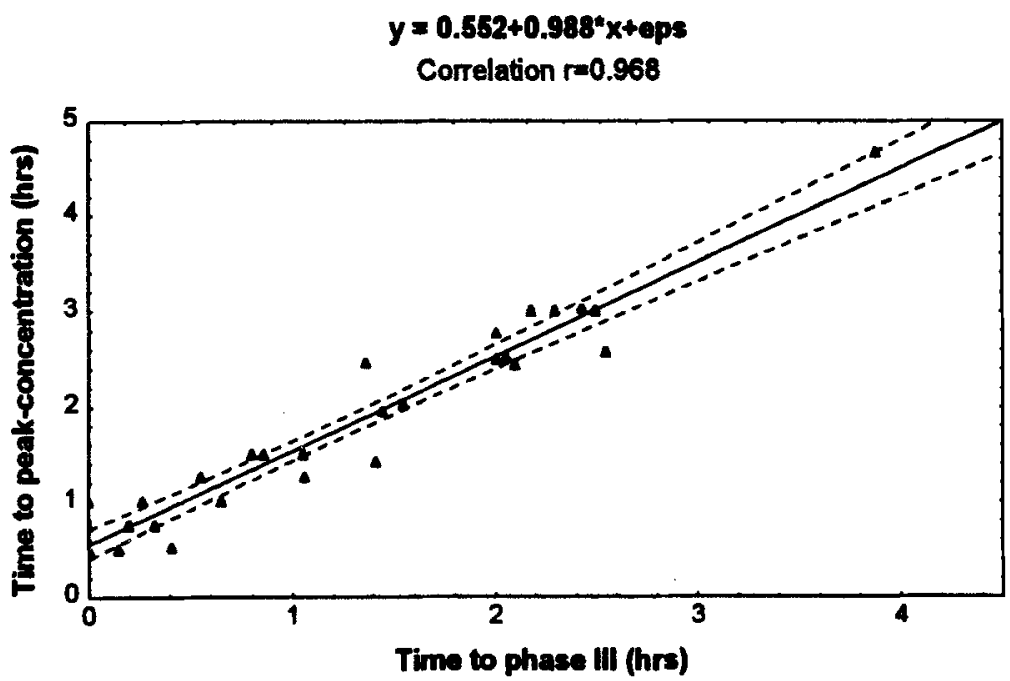

FIG. 7. Correlation between time to Phase II/III (=time period between dosing and Phase II/III onset) and time to peak concentration of S-celiprolol $(n=16)$ (correlation was obtained by linear regression analysis).

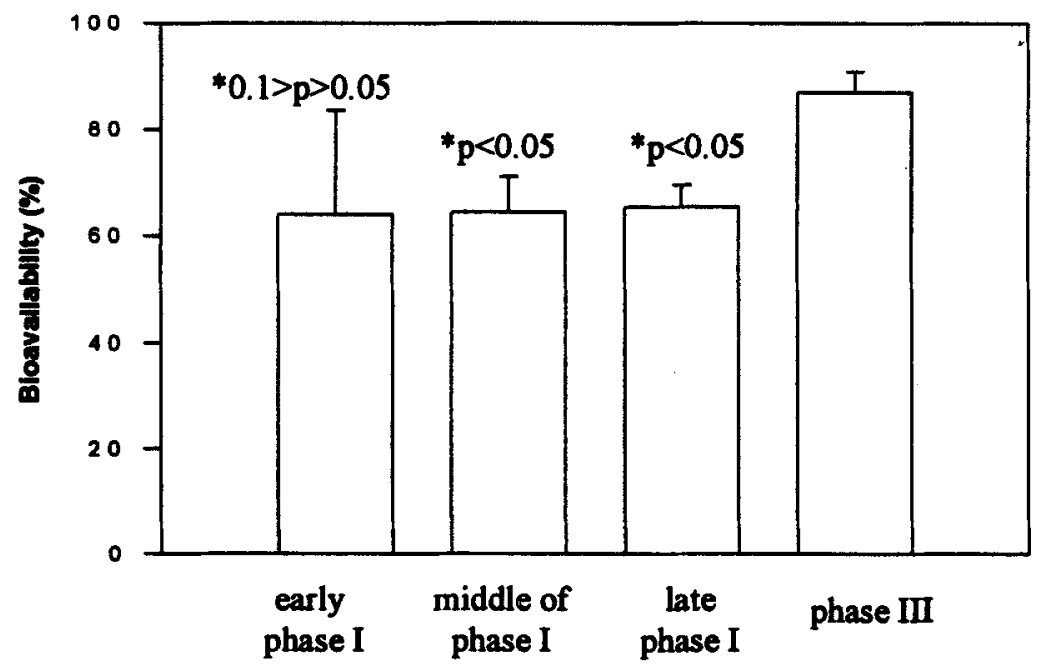

FIG. 8. Bioavailability of S-celiprolol following administration in different motility phases. 
Table I. Pharmacokinetic Parameters from Four Dogs (Noncompartmental Analysis) ${ }^{a}$

\begin{tabular}{lccccc}
\hline Parameter & Enantiomer & Dog A & Dog B & Dog C & Dog D \\
\hline$C_{\max }(\mu \mathrm{g} / \mathrm{ml})$ & $S$ & $1.49 \pm 0.24$ & $1.53 \pm 0.25$ & $2.01 \pm 0.49$ & $1.92 \pm 0.54$ \\
& $R$ & $1.54 \pm 0.23$ & $1.54 \pm 0.24$ & $2.05 \pm 0.49$ & $1.95 \pm 0.54$ \\
$t_{\max }(\mathrm{hr})$ & $S$ & $0.81 \pm 0.38$ & $1.56 \pm 1.01$ & $0.94 \pm 0.38$ & $1.06 \pm 0.43$ \\
& $R$ & $0.81 \pm 0.38$ & $1.56 \pm 1.01$ & $0.94 \pm 0.38$ & $1.06 \pm 0.43$ \\
$A U C\left(\mu \mathrm{g}^{*} \mathrm{hr} / \mathrm{ml}\right)$ & $S$ & $2.90 \pm 0.51$ & $3.94 \pm 0.84$ & $4.09 \pm 0.29$ & $4.54 \pm 0.76$ \\
& $R$ & $3.11 \pm 0.64$ & $4.06 \pm 0.87$ & $4.59 \pm 0.40$ & $5.02 \pm 0.78$ \\
$A U M C\left(\mu \mathrm{g}^{*} \mathrm{hr}^{2} / \mathrm{ml}\right)$ & $S$ & $9.97 \pm 1.83$ & $6.18 \pm 2.5$ & $14.25 \pm 2.03$ & $17.62 \pm 4.49$ \\
& $R$ & $10.97 \pm 3.07$ & $16.78 \pm 2.1$ & $17.43 \pm 2.88$ & $21.45 \pm 5.97$ \\
$k_{\text {el }}\left(\mathrm{hr}^{-1}\right)$ & $S$ & $0.31 \pm 0.03$ & $0.33 \pm 0.06$ & $0.30 \pm 0.04$ & $0.29 \pm 0.05$ \\
& $R$ & $0.30 \pm 0.04$ & $0.32 \pm 0.05$ & $0.28 \pm 0.05$ & $0.26 \pm 0.05$ \\
$t_{1 / 2}(\mathrm{hr})$ & $S$ & $2.29 \pm 0.23$ & $2.18 \pm 0.40$ & $2.39 \pm 0.32$ & $2.46 \pm 0.42$ \\
& $R$ & $2.30 \pm 0.29$ & $2.24 \pm 0.37$ & $2.58 \pm 0.42$ & $2.72 \pm 0.54$ \\
$M R T(\mathrm{hr})$ & $S$ & $3.43 \pm 0.21$ & $4.16 \pm 0.62$ & $3.48 \pm 0.34$ & $3.89 \pm 0.85$ \\
& $R$ & $3.48 \pm 0.48$ & $4.22 \pm 0.54$ & $3.78 \pm 0.34$ & $4.25 \pm 1.03$ \\
$M A T(\mathrm{hr})$ & $S$ & $1.27 \pm 0.21$ & $2.07 \pm 0.62$ & $1.16 \pm 0.34$ & $0.87 \pm 0.79$ \\
& $R$ & $1.38 \pm 0.48$ & $2.13 \pm 0.54$ & $1.24 \pm 0.35$ & $1.29 \pm 1.03$ \\
$C L(\mathrm{I} / \mathrm{hr})$ & $S$ & $26.37 \pm 4.07$ & $19.65 \pm 3.88$ & $18.41 \pm 1.32$ & $16.91 \pm 2.82$ \\
& $R$ & $24.98 \pm 5.97$ & $19.17 \pm 4.07$ & $16.43 \pm 1.42$ & $15.23 \pm 2.43$ \\
$V_{\text {dis }}(1)$ & $S$ & $59.41 \pm 8.55$ & $34.51 \pm 8.01$ & $53.93 \pm 13.1$ & $40.42 \pm 5.78$ \\
& $R$ & $59.99 \pm 7.51$ & $36.95 \pm 6.52$ & $50.94 \pm 7.29$ & $45.92 \pm 7.27$ \\
Bioavailability $(\%)$ & $S$ & $68.58 \pm 12.07$ & $62.65 \pm 13.39$ & $74.90 \pm 5.28$ & $73.40 \pm 12.28$ \\
& $R$ & $73.53 \pm 15.13$ & $61.73 \pm 13.3$ & $79.60 \pm 6.97$ & $75.08 \pm 11.73$ \\
\hline
\end{tabular}

${ }^{a}$ Mean \pm SD from four po treatments with $150 \mathrm{mg}$ racemic celiprolol- $\mathrm{HCl}$ as a solution.

the quiescent phase, because of concentration differences depending on the time to Phase II/III due to dilution as mentioned earlier. Differences in drug concentration might also explain the variations in bioavailability (45-90\%), which could reflect active intestinal secretion or an interaction with bile salts.

The average individual pharmacokinetic parameters, the mean parameters for all four dogs, and the average population pharmacokinetic parameters estimated by NONMEM are given in Table II. Individual fits for iv and po data are shown in Fig. 9( $a$ and $b$ ), supporting the ability of the twophase absorption model to predict lag times and multiple peaks in the plasma concentration-time profile of celiprolol in dogs, when the motility parameters are known.

In the first step, the parameters of the model were fitted simultaneously to four sets of oral data combined with the corresponding intravenous data. The values for the pharmacokinetic parameters within one dog, as well as the mean values for all four dogs were acceptably tight. The standard errors for the parameter estimates amounted to $10-20 \%$ for the individuals and $10-30 \%$ for the mean values. Especially, the initial absorption rate constant with $2.8 \pm 12 \mathrm{hr}^{-1}$ was surprisingly low in its variation considering the small number of sample points $(n=2-4)$ during the absorption phase. The mean 
Table II. NONMEM Pharmacokinetic Parameter Estimates of the Individual and Population Analysis of S-Celiprolol $(n=4)$

\begin{tabular}{lrccccc}
\hline & \multicolumn{1}{c}{ Dog A } & Dog B & Dog C & Dog D & Mean & Population \\
\hline$K_{\mathrm{a} 1}\left(\mathrm{hr}^{-1}\right)$ & $3.1 \pm 0.42$ & $2.56 \pm 0.42$ & $2.73 \pm 0.45$ & $2.9 \pm 00.9$ & $2.8 \pm 0.12$ & $2.54 \pm 0.35$ \\
$K_{\mathrm{az}}\left(\mathrm{hr}^{-1}\right)$ & $0.67 \pm 0.57$ & $0.33 \pm 0.2$ & $0.31 \pm 0.12$ & $0.23 \pm 0.69$ & $0.45 \pm 0.1$ & $0.23 \pm 0.04$ \\
$K_{34}\left(\mathrm{hr}^{-1}\right)$ & $4.6 \pm 0.67$ & $12.8 \pm 0.86$ & $7.01 \pm 0.79$ & $9.85 \pm 3.79$ & $8.57 \pm 1.77$ & $9.33 \pm 1.07$ \\
$K_{43}\left(\mathrm{hr}^{-1}\right)$ & $1.0 \pm 0.16$ & $1.96 \pm 0.22$ & $1.36 \pm 0.18$ & $1.93 \pm 0.94$ & $1.53 \pm 0.23$ & $2.1 \pm 0.21$ \\
$K_{30}\left(\mathrm{hr}^{-1}\right)$ & $2.64 \pm 0.31$ & $5.37 \pm 0.52$ & $3.05 \pm 0.25$ & $3.99 \pm 0.81$ & $3.75 \pm 0.61$ & $4.46 \pm 0.31$ \\
$V(\mathrm{~L})$ & $7.26 \pm 0.82$ & $2.38 \pm 0.07$ & $5.06 \pm 0.51$ & $4.26 \pm 1.02$ & $4.71 \pm 1.01$ & $4.05 \pm 0.68$ \\
$t_{\text {abs1 }}(\mathrm{hr})$ & $0.07 \pm 0.01$ & $0.44 \pm 0.01$ & $0.1 \pm 0.02$ & $0.37 \pm 0.05$ & $0.24 \pm 0.09$ & $0.13 \pm 0.03$ \\
$F 1$ & $0.63 \pm 0.04$ & $0.47 \pm 0.03$ & $0.76 \pm 0.04$ & $0.74 \pm 0.12$ & $0.65 \pm 0.07$ & $0.73 \pm 0.07$ \\
$t_{\text {abs2 }}(\mathrm{hr})$ & - & $0.86 \pm 0.05$ & $0.97 \pm 0.12$ & $0.84 \pm 0.05$ & $0.58 \pm 0.22$ & $0.43 \pm 0.03$ \\
$F 2$ & $0.11 \pm 0.04$ & $0.21 \pm 0.1$ & $0.07 \pm 0.01$ & $0.26 \pm 0.05$ & $0.15 \pm 0.04$ & $0.09 \pm 0.01$ \\
$M$ VOF & -298.1 & -151.51 & -188.26 & -111.17 & -187.26 & -596.30 \\
\hline
\end{tabular}

${ }^{a}$ All values are means $\pm S E$.

${ }^{b}$ Minimum value of objective function.

bioavailability $(F I+F 2)$ from the individual analysis was $0.8 \pm 0.11$, which was in good agreement with data obtained from the noncompartmental analysis with $0.7 \pm 0.03$ (Table 1).

In the second step, 4 iv data sets and subsequently 16 sets of oral data together with the four corresponding intravenous data sets were analyzed in the population model. Figure 10a shows the NONMEM population fit for the iv data and Fig. 10b the population fit of the entire data pool. In most cases, the population pharmacokinetic parameter estimates exhibit less variability than the mean values from the individual analysis (Table II). This is due to the ability of NONMEM to evaluate intrasubject and intersubject variations simultaneously. The individual analysis considers only intrasubject variations, therefore resulting in mean values from this step that are less precise than the population parameter estimates. Furthermore, the larger number of data points in the population analysis provides NONMEM with more information to obtain accurate estimates. The goodness-of-fit from the estimation step is represented by the minimum value of objective function ( $M V O F)$, which is comparable to the sum of squares in regression analysis. A drop in the $M V O F$ reflects therefore an improvement of the model. The overall population bioavailability estimated $(F I+F 2)$ was $0.82 \pm 0.08$, which was in good agreement with the average individual bioavailability estimated in four dogs $(0.8 \pm 0.11)$. Approximately $10 \%$ of the orally available dose was assigned to the second peak when the entire data pool of 16 po experiments was taken into consideration. Intrasubject variability was calculated to be $17 \%$, and the interindividual variability for bioavailability was found to be $29 \%$. 
a)

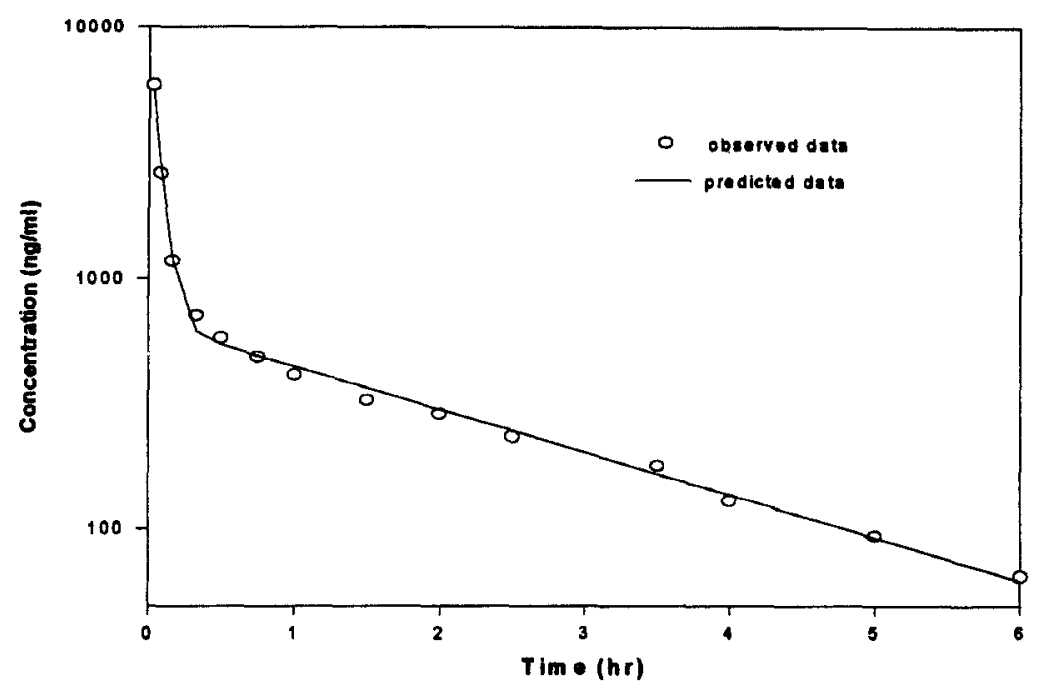

b)

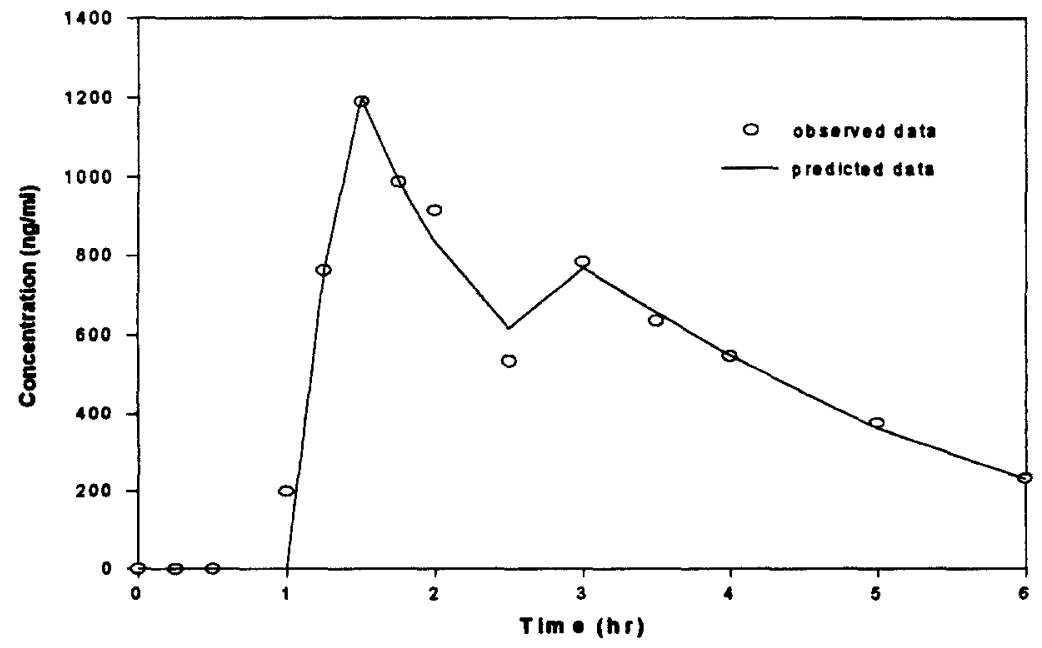

F1G. 9. NONMEM individual fit of (a) iv data of S-celiprolol (Dog B) and (b) po data with a double peak (Dog B). 
a)

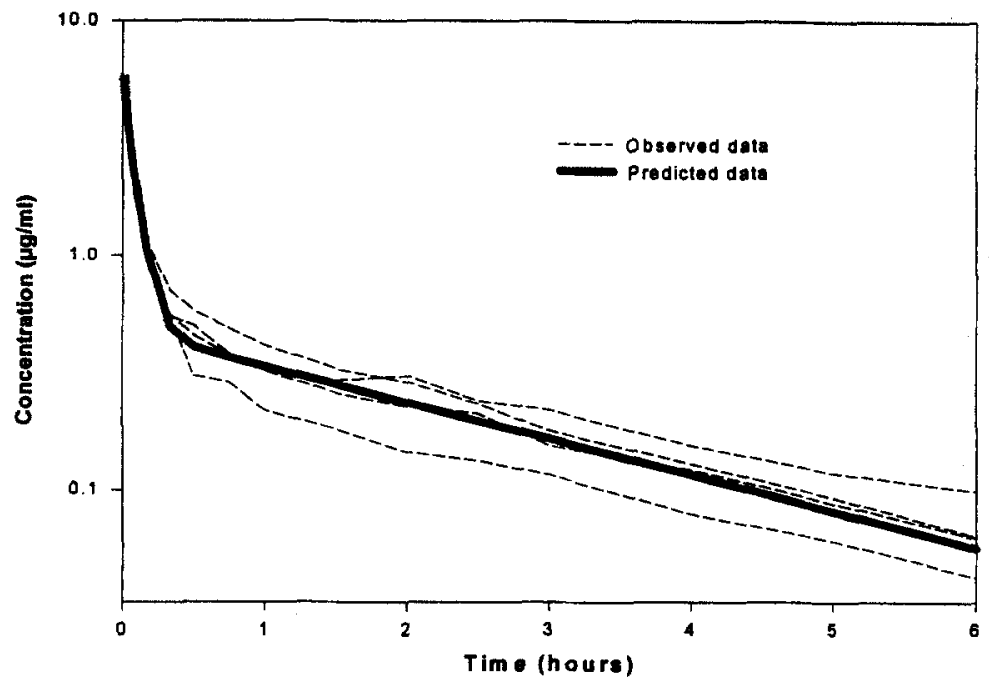

b)

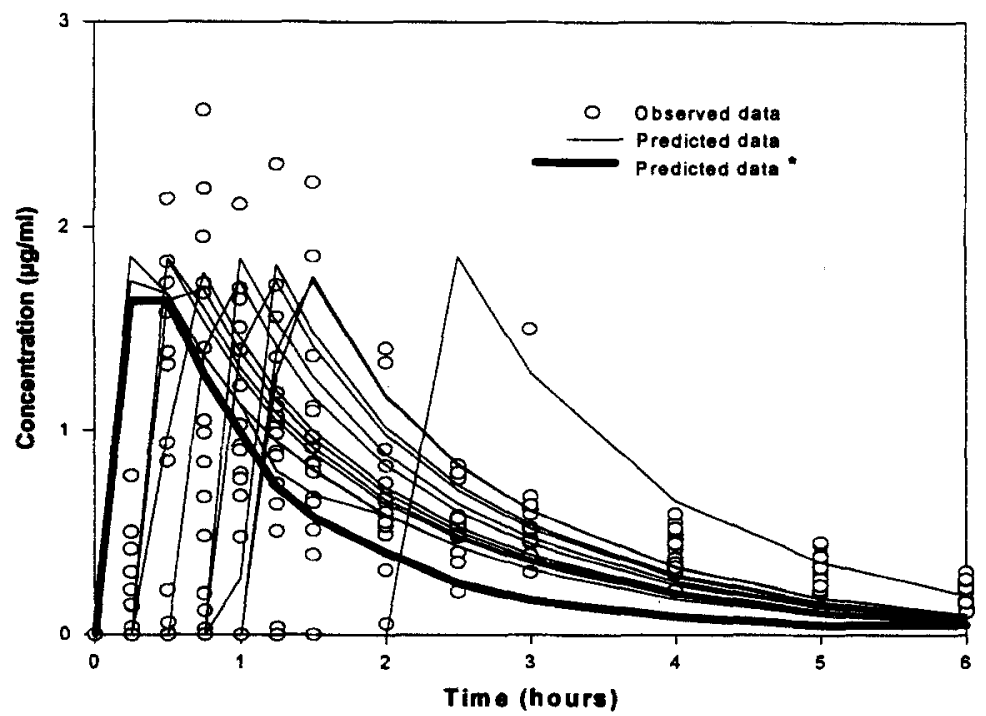

FIG. 10. NONMEM population fit of (a) iv data sets $(n=4)$ and (b) the complete po and iv data sets. Predicted data: fit obtained for the po data sets including the lag times. Predicted data*: mean population fit after subtracting the lag times represents the population curve obtained when gastric emptying occurs immediately after dosing. 


\section{DISCUSSION}

When compared to human data described earlier (3), in the dog, the first peak in the plasma concentration-time profile was more pronounced than the second one and the first input rate constant was about 5 to 10 times higher than the second one. Lower input rates for the second absorption phase may reflect the concentration-dependent uptake of celiprolol, since the drug solution undergoes dilution in the stomach, and/or might be due to the less frequent sampling time during that part of the study. The differences in the peak ratios could be explained by the fact that celiprolol was adminstered as a solution in the present study, whereas it was given as a tablet (celiprolol $200 \mathrm{mg}$, Selectol ${ }^{(3)}$ ) in the human studies (3). Administration of a solid dosage form in the fasted state may result in a partial emptying of dissolved or suspended drug particles. Thus, for the remaining part of the tablet, a passage of another active phase is required, so that the second portion of the dose reaches the absorption site. Or parts of the dose might even remain in the stomach during a short active phase, and only be emptied with the occurrence of the following Phase II/III. Similar observations were reported in a flurbiprofen study (19), where the rate and extent of absorption during the first phase decreased when the drug was given as a tablet, and almost $70 \%$ of the total drug absorption was associated with the second absorption phase. Furthermore, in all clinical studies with celiprolol the subjects had ingested a meal $0.5-3 \mathrm{hr}$ after drug intake, which terminates cyclic gastric emptying for various lengths of time and makes a comparison between the data even more difficult.

The double-peak pattern observed in the dog is most likely due to gastric emptying. This is further supported by the observation that the plasma halflife in dogs $(2-2.5 \mathrm{hr})$ is shorter than in humans $(4-5 \mathrm{hr})$, which makes the influence of gastric emptying as the rate-limiting step for absorption more apparent. Simulation studies have shown that an increase in the predominant elimination rate constant results in a significant increase in the frequency of double peaks (31). Amidon et al. tried to provide an additional explanation for the discontinuous plasma profile of cimetidine by taking the intestinal transit rate into consideration. They concluded that dosing time relative to phasic activity together with variability in flow rate out of the stomach are crucial to the occurrence of double peaks $(16,31)$. Biphasic gastric emptying was also shown to be one causative factor of the double-peak behaviour for acetaminophen (15).

The increased bioavailability of celiprolol when the drug was administered during Phase II/III of the gastrointestinal cycle could be a function of increased bile salt levels in the intestine due to gallbladder-emptying during Phase II/III (32). However, for $\beta$-blockers there is evidence for a decrease 
in absorption in the presence of bile salts due to formation of insoluble complexes as reported for nadolol and pafenolol $(32,33)$, but absorption studies with celiprolol in rats with and without bile salts performed by our group did not show any evidence for this interaction (data not shown). Furthermore, for most drugs bile salts function as solubilization enhancers, but since celiprolol exhibits very high aqueous solubility, no major effect is expected from the presence of bile salts. The contribution of bile to the increased absorption in Phase II/III appears therefore to be of minor importance. An increase in transit time might lead to an increased rate of absorption due to more rapid gastric emptying as well as a decrease in extent of absorption as a consequence of reduced contact time with the small intestinal surface area. For celiprolol a decrease in time to gastric emptying might in part contribute to the increased bioavailability. Although data showing the actual mechanism of this observation is not readily available yet, the most likely explanation appears to be that differences in intestinal concentration of celiprolol caused by more rapid gastric emptying and less dilution by the gastrointestinal fluids account for the increased bioavailability, since celiprolol exhibits nonlinear increase in oral absorption with increasing doses.

With respect to the population model, fitting the data to the twocompartment model with two lag times without incorporating the parameter "time to Phase III" resulted in an unsuccessful minimization, because the second absorption phase was not appropriately fitted anymore. The gastrointestinal motility data, incorporated as a covariant for the lag time into the population pharmacokinetic model, therefore improves the precision of the plasma concentration predictions significantly.

In conclusion, the two-absorption phase open model incorporating intestinal motility data was shown to be appropriate in predicting bimodal plasma concentration-time profiles of celiprolol. The observed double-peak phenomenon in dogs is most likely due to variations in gastric emptying. However, the shape and frequency of the double peaks of celiprolol in dogs differ from data obtained in humans, which was also observed for cimetidine (34). Therefore, secretion of celiprolol into the intestinal lumen $(27,35)$ mediated by membrane-bound p-glycoprotein or a proton/cation transporter might play a role with regard to double-peak formation and nonlinear bioavailability. Saturation of the transport system with high drug concentrations would result in enhanced absorption and, therefore, increased bioavailability. Assuming a site-specific location of the transport system in the proximal intestine, a second absorption phase might occur in the lower intestine, leading to a second peak in the plasma profile. Very recent studies in Caco-2 cells as well as clinical studies with talinolol, a $\beta$-blocker structurally close to celiprolol, performed by Wetterich et al. 
(36) have shown further evidence for this class of compounds being actively secreted into the intestine.

Hence, the origin of the variations in human data might be more complex and only in part be explained by the gastrointestinal motility patterns.

\section{REFERENCES}

1. R. J. Milne and M. T. Buckley. Celiprolol: An undated review of its pharmacokinetic properties and therapeutic efficacy in cardiovascular diseases. Drugs 41:941-969 (1991).

2. C. Hartmann, D. Krauss, H. Spahn, and E. Mutschler. Simultaneously determination of (R)- and (S)-celiprolol in human plasma and urine: High performance liquid chromatographic assay on a chiral stationary phase with fluorimetric detection. J. Chromatog. 496:387-396 (1989).

3. C. Hartmann. Enantiomerenanalytik und -kinetik des $\beta$-Adrenozeptorenblockers Celiprolol. Ph.D. thesis, Department of Pharmacology, University of Frankfurt, Germany 1989.

4. R. D. Smith, and P. S. Wolf. Celiprolol. In A. Sciabine (ed.) New Drugs Annual: Cardiovascular Drugs, Vol. 2, Raven, New York, 1984.

5. J. R. Norris, E. H. Lee, D. Muirhead, and S. W. Saunders. A pharmacokinetic evaluation of celiprolol in healthy elderly volunteers. $J$. Cardiovasc. Pharmacol. 8:S91S92 (1986).

6. R. F. Bergstrom, D. R. Kay, and J. G. Wagner. The pharmacokinetics of penicillamine in a female mongrel dog. J. Pharmacokin. Biopharm. 9:603-621 (1981).

7. C. G. Regárdh, A. Heggelund, K. Kylberg-Hanssen, and P. Lundborg. Pharmacokinetics of pafenolol after iv and oral administration of three separate doses of different strength to man. Biopharm. Drug Disp. 11:607-617 (1990).

8. H. Lennernäs, and C. G. Regårdh. Pharmacokinetics of pafenolol in the rat: A suitable model for studying absorption mechanisms of a drug exhibiting unusual absorption properties in man. Biopharm. Drug Disp. 11:619-631 (1990).

9. T. Yamagushi, C. Ikeda, and Y. Sekine. Intestinal absorption of a $\boldsymbol{\beta}$-adrenergic blocking agent nadolol I. Comparison of absorption behaviour of nadolol with those of other $\beta$-blocking agents in rats. Chem. Pharm. Bull. 34:3362-3369 (1986).

10. F. S. Caruso, H. D. Doshan, P. H. Hernandez, R. Costello, W. Applin, and E. S. Neiss. Celiprolol: Pharmacokinetics and duration of pharmacodynamic activity. $B r . J$. Clin. Pract. 40:12-16 (1985).

11. J. Dreyfuss, D. L. Griffith, S. M. Singhvi, J. M. Shaw, and J. J. Ross, Jr. Pharmacokinetics of nadolol, a beta-receptor antagonist: Administration of therapeutic singleand multiple-dosage regimens to hypertensive patients. J. Clin. Pharmacol. 19:712-720 (1979).

12. T. Yamagushi, C. Ikeda, and Y. Sekine. Intestinal absorption of a $\beta$-adrenergic blocking agent nadolol II. Mechanism of the inhibitory effect on the intestinal absorption of nadolol by sodium cholate in rats. Chem. Pharm. Bull. 34:3836-3843 (1986).

13. H. Lennernäs and C. G. Regårdh. Evidence for an interactions between the $\beta$-blocker pafenolol and bile salts in the intestinal lumen of the rat leading to dose-dependent oral absorption and double peaks in the plasma concentration-time profile. Pharm. Res. 10:879-883 (1993).

14. H. Ogata, N. Aoyagi, K. Kaniwa, T. Shibazaki, A. Ejima, N. Takasugi, E. Mafune, T. Hayashi, and K. Suwa. Bioavailability of nalidixic acid from uncoated tablets in humans - Part 1: Correlation with the dissolution rates of tablets. Int. J. Clin. Pharmacol. Ther. Toxicol. 22:175-183 (1984).

15. J. A. Clements, R. C. Heading, W. S. Nimmo, and L. F. Prescott. Kinetics of acetaminophen absorption and gastric emptying in man. Clin. Pharmacol. Ther. 24:420431 (1978). 
16. R. Oberle, and G. L. Amidon. The influence of variable gastric emptying and intestinal transit rates on the plasma level curve of cimetidine; an explanation for the double peak phenomenon. J. Pharmacokin. Biopharm. 15:529-544 (1987).

17. G. Mullersmann, V. P. Gotz, W. L. Russel, and H. Derendorf. Lack of clinically significant in vivo and in vitro interaction between ranitidine and sucralfate. J. Pharm. Sci. 75:995-998 (1986).

18. A. Grahnen, M. Hammarlund, and T. Lundquist. Implications of intraindividual variability in bioavailability studies of furosemide. Eur. J. Pharmacol. 27:595-602 (1984).

19. J. B. Dressman, R. R. Beradi, G. H. Elta, T. M. Gray, P. A. Montgomery, H. S. Lau, K. L. Pelekoudas, H. S. Szpunar, and J. G. Wagner. Absorption of flurbiprofen in the fed and fasted state. Pharm. Res. 9:901-907 (1992).

20. H. Lennernäs, L. Renberg, K. J. Hoffmann, C. G. Regårdh. Presystemic elimination of the beta-blocker pafenolol in the rat after oral and intraperitoneal administration and identification of a main metabolite in both rats and humans. Drug Metab. Dispos. 21:435-440 (1993).

21. Y. Plusquellec, G. Campistron, S. Staveris, J. Barre, L. Jung, J. P. Tillement, and G. Houin. A double-peak phenomenon in the pharmacokinetics of veralipride after oral administration: a double-site model for drug absorption. J. Pharmacokin. Biopharm. 15:225-239 (1987).

22. B. Liebmann, R. Ding, E. Weber, E. Mutschler, H. Spahn-Langguth, and P. Langguth. Biopharm. Drug. Disp., submitted. (1994).

23. B. Voith, H. Spahn-Langguth, R. Paliege, H. Knauf, and E. Mutschler. Etacrynic acid: Evidence for active metabolites. Naunyn-Schmiedenberg's Arch. Pharmakol. 349(Suppl) :594 (Abstract) (1994).

24. A. F. Parr, R. M. Beihn, R. M. Franz, G. J. Szpunar, and M. Jay. Correlation of ibuprofen bioavailability with gastrointestinal transit by scintigraphic monitoring of ${ }^{171}$ Er-labeled sustained release tablets. Pharm. Res. 4:486-491 (1987).

25. H. Spahn-Langguth, G. Hahn, W. Moehrke, and P. Langguth. Variabilities and discontinuities in tranylcypromine input profiles following oral dosage. Eur. J. Pharm. Sci. 2:160 (1994).

26. C. Hartmann, M. Fröhlich, D. Krauss, H, Spahn, H. Knauf, and E. Mutschler. Comparative enantioselective pharmacokinetic studies of celiprolol in healthy volunteers and patients with impaired renal function. Eur. J. Clin. Pharmacol. 38:573-576 (1990).

27. J. Karlson, S. M. Kuo, J. Ziemniak, and P. Artursson. Transport of celiprolol across human intestinal epithelial (Caco-2) cells: Mediation of secretion by multiple transporters including P-glycoprotein. Br. J. Pharmacol. 110:1009-1016 (1993).

28. A. J. Boeckmann, L. B. Sheiner, and S. L. Beal. NONMEM Users Guide-Park V, Introductory Guide, NONMEM project group, C255, University of California at San Francisco, 1991.

29. C. F. Code, and J. A. Marlett. The interdigestive myo-electric complex of the stomach and small bowel of dogs. J. Physiol. 246:289-309 (1975).

30. P. Kerlin, and S. Phillips. Variability of motility of the ileum and jejunum in healthy volunteers. Gastroenterology 82:694-700 (1982).

31. P. Langguth, K. M. Lee, H. Spahn-Langguth, and G. L. Amidon. Variable gastric emptying and discontinuities in drug absorption profiles: Dependence of rates and extent of cimetidine absorption on motility phases and pH. Biopharm. Drug Disp. 15:719-746 (19S4).

32. T. Yamagushi, C. Ikeda, and $Y$. Sekine. Intestinal absorption of a $\beta$-adrenergic blocking agent nadolol II. Mechanism of the inhibitory effect on the intestinal absorption of nadolol by sodium cholate in rats. Chem. Pharm. Bull. 34:3836-3843 (1986).

33. H. Lenneräs, and C. G. Regårdh. Evidence for an interaction between the $\beta$-blocker pafenolol and bile salts in the intestinal lumen of the rat leading to dose-dependent oral absorption and double peaks in the plasma concentration-time profile. Pharm. Res. 10:879-883 (1993).

34. Y. Hayashi, I. Lee, and G. L. Amidon. Effects of gastrointestinal motility and pH variation on oral absorption of cimetidine in dogs. Pharm. Res. 10(Suppl):S371 (1993). 
35. S. M. Kou, B. R. Whitby, P. Artursson, and J. A. Ziemniak. The contribution of intestinal secretion to the dose-dependent absorption of celiprolol. Pharm. Res. 11:743749 (1994).

36. U. Wetterich, E. Mutschler, H. Spahn-Langguth, and P. Langguth. Evidence for intestinal secretion of the $\beta$-adrenoceptor antagonist talinolol: Data from humans and studies with Caco-2 cells. Naunyn-Schmiedenberg's Arch. Pharmakol. (Suppl.) Abstract accepted. 